\title{
New Lyapunov-Krasovskii stability condition for uncertain linear systems with interval time-varying delay Weifeng Zhang ${ }^{1, \mathrm{a}}$, Junjun $\mathrm{Hui}^{2, \mathrm{~b}}$ and Wenqi Gao ${ }^{1, \mathrm{c}}$ \\ ${ }^{1}$ Lanzhou Institute of Technology, Lanzhou 730050, China \\ ${ }^{2}$ Mailbox 150 extension 11, Baoji, Shaanxi 721013, China ${ }^{3}$ \\ a zhangwf138@126.com, ${ }^{\mathrm{b}}$ ep22stone@163.com, ${ }^{\mathrm{C}}$ gaowenqi@163.com
}

\begin{abstract}
Keywords: L-K functional; delay decomposition; Distributed delay; Linear matrix inequality (LMI) Abstract. This paper investigates the robust delay-dependent stability problem of a class of linear uncertain system with interval time-varying delay. Based on delay-central point method, the whole delay interval is divided into two equidistant subintervals at its central point and a new Lyapunov-Krasovskii (L-K) functionals which contains some triple-integral terms and augment terms are introduced on these intervals. Then, by using L-K stability theorem, integral inequality method and convex combination technique, a new delay-dependent stability criteria for the system is formulated in terms of linear matrix inequalities (LMIs). Unlike existing methodologies, when bounding the cross-terms that emerge from the time derivative of the L-K functional, neither superfluous free weighting matrices are introduced nor any useful terms are neglected, only using tighter integral inequalities and a very few free weighting matrices for express the relationship of the correlative terms, so that it can reduce the complexity both in theoretical derivation and in computation. Finally, numerical examples are given to illustrate the effectiveness and an improvement over some existing results in the literature with the proposed results.
\end{abstract}

\section{Introduction}

Time delay is encountered in many dynamic systems such as chemical or process control systems and networked control systems [1].Time delay is always one of the sources of instability and poor performance. Hence, stability analysis and stabilization of system with time-delays have received considerable attention in the past few years[2-13].Very recently, systems with time-varying delay in a known interval have been studied in [2,6-13], wherein the time-delay may vary in a range for which the lower bound is not restrict to being zero.

In view of the stability analysis for the systems with interval time-varying delay, the existing stability criteria are usually classified into two major categories, namely, delay-independent ones, and delay-dependent ones. Generally speaking, the delay-dependent stability criterion is less conservative than delay-independent stability when the time-delay is small. A general framework for stability analysis is Lyapunov-Krasovskii functional combine with the Linear matrix inequality. Under this framework, an important issue is to enlarge the feasible region of stability criteria. As for the analysis method, there were free-weighting matrix method[2],augmented Lyapunov functional approach[3] and delay partitioning approach[5,6,8] etc., the common advantages of the above method is the delay information can be fully used, which avail reduce the conservatism, however, it can also lead to the increase of computational complexity since many slack variables are introduced. Jensen's integral inequality approach is another important method, this approach possesses few variables characteristics, so can provide a simple form of stability conditions. Gu[1] was the first one to introduced this method to the stability analysis of time-delay systems. Soon afterwards, many researchers, such as Zhang et al. [8],Shao et al.[10], Ramakrishnan and Ray[9,13] further extended the Jensen's integral inequality to some new forms and obtained some less conservative results. Nevertheless, there still exists room for further improvements.

Motivated by the above discussions, we further discuss the stability of uncertain linear systems with interval time-varying delays. Based on delay-central point method, the whole delay interval is 
divided into two equidistant subintervals at its central point and a new L-K functionals which contains some triple-integral terms and augment terms are introduced on these intervals. Then, by using L-K stability theorem, integral inequality method and convex combination technique, a new delay-dependent stability criteria for the system is formulated in terms of linear matrix inequalities (LMIs). Numerical examples are given to illustrate the effectiveness and less conservatism of the proposed method.

\section{Problem description and preliminaries}

Notations: Throughout this paper, $\boldsymbol{R}^{n}$ denotes the n-dimensional Euclidian space, $\boldsymbol{R}^{n \times m}$ is the set of $n \times m$ real matrice, $X>0$, for $X \in \boldsymbol{R}^{n \times m}$, means that the matrix $X$ is a real symmetric positive definite. For an arbitrary matrix $B$ and two symmetric matrices $A$ and $C,\left[\begin{array}{ll}A & B \\ * & C\end{array}\right]$ denotes a symmetric matrix, where $*$ denotes the entries implied by symmetry, $e_{i}$ represents block entry matrices of appropriate dimensions, i.e., if $i=1,2,3$,then,

$$
e_{1}=\left[\begin{array}{lll}
I & 0 & 0
\end{array}\right], e_{2}=\left[\begin{array}{lll}
0 & I & 0
\end{array}\right], e_{3}=\left[\begin{array}{lll}
0 & 0 & I
\end{array}\right] \text {. }
$$

Consider the following linear uncertain system with time-varying delay

$$
\left\{\begin{array}{l}
x(t)=(A+\Delta A(t)) x(t)+(B+\Delta B(t)) x(t-h(t)) \\
x(t)=\varphi(t), t \in\left[-h_{2}, 0\right]
\end{array}\right.
$$

Where, $x(t) \in \boldsymbol{R}^{n}$ is the state vector, $A, B$ are constant matrices with appropriate dimensions, $h(t)$ is a time-varying continuous function, satisfy the following two scenarios conditions:

Case1: $0 \leq h_{m} \leq h(t) \leq h_{M}, \hat{h}(t) \leq \mu, \forall t \geq 0$;

Case2: $0 \leq h_{m} \leq h(t) \leq h_{M}, \forall t \geq 0$

$\Delta A(t), \Delta B(t)$ denote parameter uncertainties satisfying the following condition:

$$
[\Delta A(t) \quad \Delta B(t)]=D F(t)\left[\begin{array}{ll}
E_{a} & E_{b}
\end{array}\right]
$$

where, $D, E_{a}, E_{b}$ are constant matrices with appropriate dimensions; and $F(t)$ is an unknown time-varying matrix , which is Lebesque measurable in $t$ and satisfies $F(t)^{\mathrm{T}} F(t) \leq I, \forall t$.

The following lemma is introduced which has an important role in the derivation of the main results.

Lemma 1[8]. For any constant matrix $R \in \boldsymbol{R}^{n \times n}$, scalar $h>0$, and vector function $(t):[0, h] \rightarrow \boldsymbol{R}^{\mathrm{n}}$ such that the integration $-h \int_{t-h}^{0} x \&(s) R(s) d s$ is well defined, then the following inequality holds:

$$
-h \int_{t-h}^{0} \boldsymbol{R}(s) \boldsymbol{R} \delta(s) d s \leq\left[\begin{array}{c}
\boldsymbol{x}(t) \\
\boldsymbol{x}(t-h)
\end{array}\right]^{T}\left[\begin{array}{cc}
-\boldsymbol{R} & \boldsymbol{R} \\
\boldsymbol{R} & -\boldsymbol{R}
\end{array}\right]\left[\begin{array}{c}
\boldsymbol{x}(t) \\
\boldsymbol{x}(t-h)
\end{array}\right]
$$

Lemma 2[9]. For any constant matrix $R \in \boldsymbol{R}^{n \times n}, R=R^{T}>0$, scalars $h_{1} \leq h(t) \leq h_{2}$, and vector function $f(t):\left[-h_{2},-h_{1}\right] \rightarrow \boldsymbol{R}^{\mathrm{n}}$ such that the integration $\int_{t-h_{2}}^{t-h_{1}} \&(t) \boldsymbol{R}(t) d t$ is well defined, then the following inequality holds:

$$
-\left(h_{2}-h_{1}\right) \int_{t-h_{2}}^{t-h_{1}} \&(t) \boldsymbol{R}\{t) d t \leq \xi^{T}(t)\left[\Pi_{R}+\left(h(t)-h_{1}\right) \Pi_{1, R}+\left(h_{2}-h(t)\right) \Pi_{2, R}\right] \xi(t)
$$

Where $\xi^{T}(t)=\left[\begin{array}{lll}x^{T}\left(t-h_{1}\right) & x^{T}(t-h(t)) & x^{T}\left(t-h_{2}\right)\end{array}\right]$ and 


$$
\begin{aligned}
& \Pi_{R}=\left[\begin{array}{ccc}
-R & R & 0 \\
* & -2 R & R \\
* & * & -R
\end{array}\right], \Pi_{1, R}=\left(e_{2}-e_{3}\right)^{T}\left(-\frac{R}{h_{2}-h_{1}}\right)\left(e_{2}-e_{3}\right), \\
& \Pi_{2, R}=\left(e_{1}-e_{2}\right)^{T}\left(-\frac{R}{h_{2}-h_{1}}\right)\left(e_{1}-e_{2}\right) .
\end{aligned}
$$

Lemma 3[5]. Suppose $\gamma_{1} \leq \gamma(t) \leq \gamma_{2}$, Where $\gamma():. \boldsymbol{R}_{+}\left(\right.$or $\left.\boldsymbol{Z}_{+}\right) \rightarrow \boldsymbol{R}_{+}\left(\right.$or $\left.\boldsymbol{Z}_{+}\right)$. Then,for any constant matrices $\Xi_{1}, \Xi_{2}$ and $\Omega$ with proper dimensions, the following matrix inequality

$$
\Omega+\left(\gamma(t)-\gamma_{1}\right) \Xi_{1}+\left(\gamma_{2}-\gamma(t)\right) \Xi_{2}<0
$$

holds, if and only if

$$
\Omega+\left(\gamma_{2}-\gamma_{1}\right) \Xi_{1}<0, \Omega+\left(\gamma_{2}-\gamma_{1}\right) \Xi_{2}<0
$$

Lemma 4 [14]. Given matrices $Q=Q^{T}, H$, and $E$ with appropriate dimensions, then

$$
Q+H F(t) E+E^{\mathrm{T}} F(t)^{\mathrm{T}} H^{\mathrm{T}}<0
$$

for all $F(t)$ satisfying $F(t)^{T} F(t) \leq I$, if and only if there exists a scalar $\varepsilon>0$, such that

$$
Q+\varepsilon^{-1} H H^{\mathrm{T}}+\varepsilon E^{\mathrm{T}} E<0
$$

\section{Main result}

First consider a nominal system (1):

$$
\left\{\begin{array}{l}
x(t)=A x(t)+B x(t-h(t)) \\
x(t)=\varphi(t), t \in\left[-h_{2}, 0\right]
\end{array}\right.
$$

Theorem 1 For given values of $h_{1}, h_{2}$ and $\mu$,System (5) is asymptotically stable, if there exist matrices $\left[\begin{array}{ccc}P_{11} & P_{12} & P_{13} \\ * & P_{22} & P_{23} \\ * & * & P_{33}\end{array}\right]>0, Q_{j}>0, Z_{j}>0 j=1,2, S>0$ and $\left[\begin{array}{cc}R_{11} & R_{12} \\ * & R_{22}\end{array}\right] \geq 0,\left[\begin{array}{cc}W_{11} & W_{12} \\ * & W_{22}\end{array}\right] \geq 0$ with appropriate dimensions such that the following LMIs hold

$$
\begin{aligned}
& {\left[\begin{array}{cc}
\Phi+\Phi_{i} & T^{T} M_{1} \\
* & -M_{1}
\end{array}\right]<0, i=1,2} \\
& {\left[\begin{array}{cc}
\Phi+\Phi_{i} & T^{T} M_{2} \\
* & -M_{2}
\end{array}\right]<0, i=1,2}
\end{aligned}
$$

where

$$
\begin{gathered}
{\left[\begin{array}{cccccccc}
\Phi_{11} & \Phi_{12} & P_{13} & P_{11} B & \Phi_{15} & W_{12} & \Phi_{17} & \Phi_{18} \\
* & \Phi_{22} & -R_{12} & 0 & 0 & 0 & 0 & 0 \\
* & * & \Phi_{33} & Z_{2} & 0 & 0 & 0 & 0 \\
* & * & * & \Phi_{44} & Z_{2} & 0 & B^{T} P_{12} & B^{T} P_{13} \\
* & * & * & * & \Phi_{55} & -W_{12}^{T} & \Phi_{57} & \Phi_{58} \\
* & * & * & * & * & \Phi_{66} & 0 & 0 \\
* & * & * & * & * & * & -4 Q_{1} & 0 \\
* & * & * & * & * & * & * & -4 Q_{2}
\end{array}\right],} \\
\Phi_{1}=\left(e_{3}-e_{4}\right)^{T}\left(-Z_{2}\right)\left(e_{3}-e_{4}\right), \Phi_{2}=\left(e_{4}-e_{5}\right)^{T}\left(-Z_{2}\right)\left(e_{4}-e_{5}\right), \\
\Phi_{11}=P_{11} A+A^{T} P_{11}+P_{12}+P_{12}^{T}+R_{11}+W_{11}+S-Z_{1}-4 h_{2}^{2} Q_{1}-4 h_{\delta}^{2} Q_{2}, \Phi_{12}=R_{12}+Z_{1}, \Phi_{15}=-P_{12}-P_{13}, \\
\Phi_{17}=A^{T} P_{12}+P_{22}^{T}+4 h_{2} Q_{1}, \quad \Phi_{18}=A^{T} P_{31}^{T}+P_{32}^{T}+4 h_{\delta} Q_{2}, \Phi_{22}=R_{22}-R_{11}-Z_{1}, \quad, \quad \Phi_{33}=-R_{22}-Z_{2}, \\
\Phi_{44}=-(1-\mu) S-2 Z_{2}, \Phi_{55}=-W_{22}-Z_{2}, \Phi_{57}=-P_{22}^{T}-P_{23}^{T}, \Phi_{58}=-P_{32}^{T}-P_{33}^{T}, \Phi_{66}=W_{22}-W_{11},
\end{gathered}
$$




$$
\begin{aligned}
& T=\left[\begin{array}{llllllll}
A & 0 & 0 & B & 0 & 0 & 0 & 0
\end{array}\right], M_{1}=\left(\frac{h_{a}}{2}\right)^{2} Z_{1}+h_{\delta}{ }^{2} Z_{2}+h_{2}^{4} Q_{1}+\left(h_{2}^{2}-h_{a}^{2}\right)^{2} Q_{2}, \\
& M_{2}=\left(\frac{h_{a}}{2}\right)^{2} Z_{1}+h_{\delta}{ }^{2} Z_{2}+h_{2}^{4} Q_{1}+\left(h_{a}^{2}-h_{1}^{2}\right)^{2} Q_{2}, h_{a}=\left(h_{1}+h_{2}\right) / 2, h_{\delta}=\left(h_{2}-h_{1}\right) / 2 .
\end{aligned}
$$

Proof. Based on delay-central point approach, we dividing delay interval into two equal subintervals at the midpoint $h_{a}$, That is $\left[h_{1}, h_{a}\right]$ and $\left[h_{a}, h_{2}\right]$, if we can proof that theorem 1 holds for the two subintervals, then theorem 1 is true.

Case 1 : when $h_{a} \leq h(t) \leq h_{2}$, Construct a L-K functional candidate as

$$
\begin{aligned}
& V(t)=V_{1}(t)+V_{2}(t)+V_{3}(t)+V_{4}(t) \\
& V_{1}(t)=\varsigma^{T}(t) P \varsigma(t) \text {, } \\
& V_{2}(t)=\int_{t-\frac{h_{a}}{2}}^{t} \xi_{1}^{T}(s) R \xi_{1}(s) d s+\int_{t-\frac{h_{2}}{2}}^{t} \xi_{2}^{T}(s) W \xi_{2}(s) d s+\int_{t-h(t)}^{t} x^{T}(s) S x(s) d s, \\
& \left.V_{3}(t)=\frac{h_{a}}{2} \int_{-\frac{h_{a}}{2}}^{0} \int_{t+\theta}^{t} \&(s) Z_{1} d(s) d s d \theta+\left(h_{2}-h_{a}\right) \int_{-h_{2}}^{-h_{a}} \int_{t+\theta}^{t} \&(s) Z_{2} \& s\right) d s d \theta \text {, } \\
& V_{4}(t)=2 h_{2}^{2} \int_{-h_{2}}^{0} \int_{\theta}^{0} \int_{t+\lambda}^{t} d \mathcal{Q}(s) Q_{1} \delta(s) d s d \lambda d \theta+2\left(h_{2}^{2}-h_{a}^{2}\right) \int_{-h_{2}}^{-h_{a}} \int_{\theta}^{0} \int_{t+\lambda}^{t} \mathcal{R}(s) Q_{2} d(s) d s d \lambda d \theta,
\end{aligned}
$$

Where

$$
\varsigma^{T}(t)=\left[\begin{array}{lll}
x^{T}(t) & \int_{t-h_{2}}^{t} x^{T}(s) d s & \int_{t-h_{2}}^{t-h_{a}} x^{T}(s) d s
\end{array}\right], \xi_{1}^{T}(t)=\left[\begin{array}{ll}
x^{T}(t) & x^{T}\left(t-\frac{h_{a}}{2}\right)
\end{array}\right], \xi_{2}^{T}(t)=\left[\begin{array}{ll}
x^{T}(t) & x^{T}\left(t-\frac{h_{2}}{2}\right)
\end{array}\right]
$$

The time-derivative of the L-K functional along the trajectory of (1) is given by

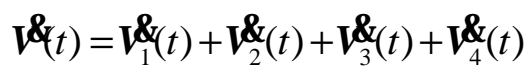

$\mathscr{V}_{1}(t)=2 \varsigma^{T}(t) P \xi(t)=2 \varsigma^{T}(t) P\left[\&(t) \quad x^{T}(t)-x^{T}\left(t-h_{2}\right) \quad x^{T}\left(t-h_{a}\right)-x^{T}\left(t-h_{2}\right)\right]^{T}$

$\boldsymbol{V}_{2}(t)=\xi_{1}^{T}(t) R \xi_{1}(t)-\xi_{1}^{T}\left(t-\frac{h_{a}}{2}\right) R \xi_{1}\left(t-\frac{h_{a}}{2}\right)+\xi_{2}^{T}(t) W \xi_{2}(t)-\xi_{2}^{T}\left(t-\frac{h_{2}}{2}\right) W \xi_{1}\left(t-\frac{h_{2}}{2}\right)+$

$$
x^{T}(t) S x(t)-(1-h(t)) x^{T}(t-h(t)) S x(t-h(t))
$$

$\mathbb{S}_{3}(t)=\left(\frac{h_{a}}{2}\right)^{2} \&(t) Z_{1} \&(t)+\left(h_{2}-h_{a}\right)^{2} \&(t) Z_{2} \&(t)-\frac{h_{a}}{2} \int_{t-\frac{h_{a}}{2}}^{t} \&(s) Z_{1} \&(s)-\left(h_{2}-h_{a}\right) \int_{t-h_{2}}^{t-h_{a}} \&(s) Z_{2} \&(s)$

$\mathbb{V}_{4}(t)=\mathbb{\&}(t)\left[h_{2}^{4} Q_{1}+\left(h_{2}^{2}-h_{a}^{2}\right)^{2} Q_{2}\right](t)-2 h_{2}^{2} \int_{-h_{2}}^{0} \int_{t+\theta}^{t} \&(s) Q_{1} \&(t) d s d \theta-2\left(h_{2}^{2}-h_{a}^{2}\right) \int_{-h_{2}}^{-h_{a}} \int_{t+\theta}^{t} \&(s) Q_{2} \&(t) d s d \theta$

From the condition (2), one can obtain:

$$
\begin{gathered}
\boldsymbol{V}_{2}(t) \leq \xi_{1}^{T}(t) R \xi_{1}(t)-\xi_{1}^{T}\left(t-\frac{h_{a}}{2}\right) R \xi_{1}\left(t-\frac{h_{a}}{2}\right)+\xi_{2}^{T}(t) W \xi_{2}(t)-\xi_{2}^{T}\left(t-\frac{h_{2}}{2}\right) W \xi_{1}\left(t-\frac{h_{2}}{2}\right)+ \\
x^{T}(t) S x(t)-(1-\mu) x^{T}(t-h(t)) S x(t-h(t))
\end{gathered}
$$

Using Lemma1,one can obtain:

$$
-\frac{h_{a}}{2} \int_{t \frac{h_{a}}{2}}^{t} \&(s) Z_{1}(s) \leq\left[\begin{array}{c}
x(t) \\
x\left(t-\frac{h_{a}}{2}\right)
\end{array}\right]^{T}\left[\begin{array}{cc}
-Z_{1} & Z_{1} \\
Z_{1} & -Z_{1}
\end{array}\right]\left[\begin{array}{c}
x(t) \\
x\left(t-\frac{h_{a}}{2}\right)
\end{array}\right]
$$

By using Lemma 2, it follows that

$$
-\left(h_{2}-h_{a}\right) \int_{t-h_{2}}^{t-h_{a}} \&(s) Z_{2}(s) \leq \xi^{T}(t)\left[\Pi_{Z_{1}}+\left(h(t)-h_{a}\right) \Pi_{1, Z_{2}}+\left(h_{2}-h(t)\right) \Pi_{2, Z_{2}}\right] \xi(t)
$$

where

$$
\xi^{T}(t)=\left[\begin{array}{lll}
x^{T}\left(t-h_{a}\right) & x^{T}(t-h(t)) & x^{T}\left(t-h_{2}\right)
\end{array}\right], \Pi_{Z_{2}}=\left[\begin{array}{ccc}
-Z_{2} & Z_{2} & 0 \\
* & -2 Z_{2} & Z_{2} \\
* & * & -Z_{2}
\end{array}\right],
$$




$$
\Pi_{1, Z_{2}}=\left(e_{2}-e_{3}\right)^{T}\left(-\frac{Z_{2}}{h_{2}-h_{a}}\right)\left(e_{2}-e_{3}\right), \Pi_{2, Z_{2}}=\left(e_{1}-e_{2}\right)^{T}\left(-\frac{Z_{2}}{h_{2}-h_{a}}\right)\left(e_{1}-e_{2}\right),
$$

Using Jensen's inequality, we have

$$
\begin{aligned}
& -\int_{-h_{2}}^{0} \int_{t+\theta}^{t} \delta(s) Q_{1}(t) d s d \theta \\
& \leq-\frac{2}{h_{2}^{2}}\left(\int_{-h_{2}}^{0} \int_{t+\theta}^{t} d(s) d s d \theta\right)^{T} Q_{1}\left(\int_{-h_{2}}^{0} \int_{t+\theta}^{t}(s) d s d \theta\right) \\
& \leq-\frac{2}{h_{2}^{2}}\left(h_{2} x(t)-\int_{t-h_{2}}^{t} x(s) d s\right)^{T} Q_{1}\left(h_{2} x(t)-\int_{t-h_{2}}^{t} x(s) d s\right) \\
& -\int_{-h_{2}}^{-h_{a}} \int_{t+\theta}^{t} \&(s) Q_{2}(t) d s d \theta \\
& \left.\leq-\frac{2}{h_{2}^{2}-h_{a}^{2}}\left(\int_{-h_{2}}^{-h_{a}} \int_{t+\theta}^{t} d s\right) d s d \theta\right)^{T} Q_{2}\left(\int_{-h_{2}}^{-h_{a}} \int_{t+\theta}^{t} x(s) d s d \theta\right) \\
& \leq-\frac{2}{h_{2}^{2}-h_{a}^{2}}\left(\left(h_{2}-h_{a}\right) x(t)-\int_{t-h_{2}}^{t-h_{a}} x(s) d s\right)^{T} Q_{2}\left(\left(h_{2}-h_{a}\right) x(t)-\int_{t-h_{2}}^{t-h_{a}} x(s) d s\right)
\end{aligned}
$$

Substituting (12) (20) in (11), the time derivative $\mathcal{L}_{t}$ ) can be expressed as follows:

$$
\left.\Phi_{t}\right) \leq \zeta_{1}^{T}(t)\left[\Phi+\left(h(t)-h_{a}\right)\left(\frac{\Phi_{1}}{h_{2}-h_{a}}\right)+\left(h_{2}-h(t)\right)\left(\frac{\Phi_{2}}{h_{2}-h_{a}}\right)+T^{T} M_{1} T\right] \zeta_{1}(t)
$$

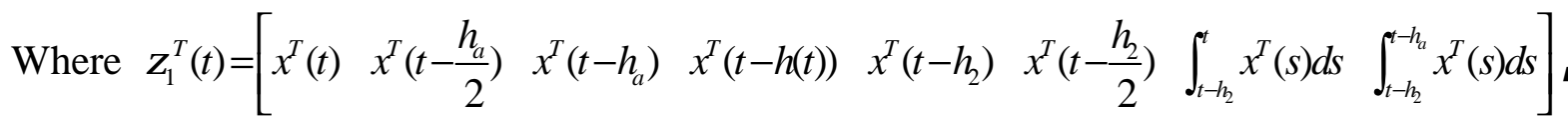

One can see that if

$$
\Phi+\left(h(t)-h_{a}\right)\left(\frac{\Phi_{1}}{h_{2}-h_{a}}\right)+\left(h_{2}-h(t)\right)\left(\frac{\Phi_{2}}{h_{2}-h_{a}}\right)+T^{T} M_{1} T<0
$$

Then, $\varepsilon_{(t)}<-\varepsilon\|x(t)\|^{2}$ for some scalar $\varepsilon>0$, from which we conclude that the nominal system (7) is asymptotically stable according to Lyapunov stability theory. From Lemma 3 and Schur compliment to (21) yield the LMI (8)

case2 : when $h_{1} \leq h(t) \leq h_{a}$, consider a L-K functional candidate as

$$
\begin{aligned}
& V(t)=V_{1}(t)+V_{2}(t)+V_{3}(t)+V_{4}(t) \\
& V_{1}(t)=\varsigma^{T}(t) P \varsigma(t), \\
& V_{2}(t)=\int_{t-\frac{h_{a}}{2}}^{t} \xi_{1}^{T}(s) R \xi_{1}(s) d s+\int_{t-\frac{h_{1}}{2}}^{t} \xi_{2}^{T}(s) W \xi_{2}(s) d s+\int_{t-h(t)}^{t} x^{T}(s) S x(s) d s, \\
& V_{3}(t)=\frac{h_{a}}{2} \int_{-\frac{h_{a}}{2}}^{0} \int_{t+\theta}^{t} \&(s) Z_{1} \delta(s) d s d \theta+\left(h_{a}-h_{1}\right) \int_{-h_{a}}^{-h_{1}} \int_{t+\theta}^{t} \&(s) Z_{2}(s) d s d \theta, \\
& \left.V_{4}(t)=2 h_{2}^{2} \int_{-h_{2}}^{0} \int_{\theta}^{0} \int_{t+\lambda}^{t} \delta(s) Q_{1} d s\right) d s d \lambda d \theta+2\left(h_{a}^{2}-h_{1}^{2}\right) \int_{-h_{a}}^{-h_{1}} \int_{\theta}^{0} \int_{t+\lambda}^{t} x(s) Q_{2} d(s) d s d \lambda d \theta,
\end{aligned}
$$

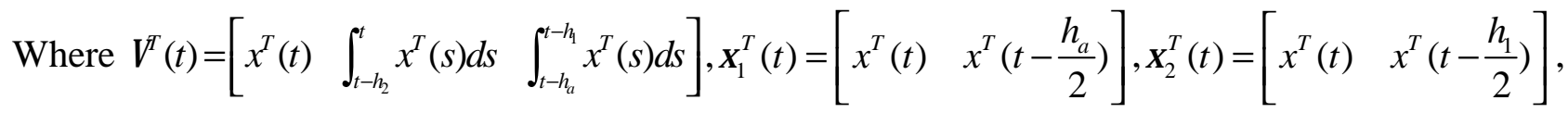
$P, R, W, S Z_{1}, Z_{2}, Q_{1}, Q_{2}$ are the same matrices used in the LK functional (10).

In the similar manner, we can obtain

$$
\mathcal{L}(t) \leq \zeta_{2}^{T}(t)\left[\Phi+\left(h(t)-h_{1}\right)\left(\frac{\Phi_{1}}{h_{a}-h_{1}}\right)+\left(h_{a}-h(t)\right)\left(\frac{\Phi_{1}}{h_{a}-h_{1}}\right)+T^{T} M_{2} T\right] \zeta_{2}(t),
$$

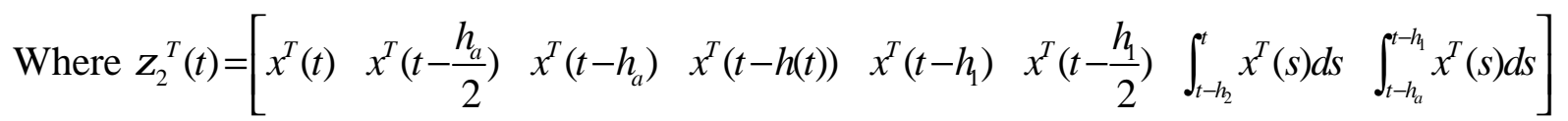


If $\Phi+\left(h(t)-h_{1}\right)\left(\frac{\Phi_{1}}{h_{a}-h_{1}}\right)+\left(h_{a}-h(t)\right)\left(\frac{\Phi_{1}}{h_{a}-h_{1}}\right)+T^{T} M_{2} T<0$

Then, system (7) is asymptotically stable. From Lemma 3 and Schur compliment to (23) yield the LMI (9), This completes the proof.

Now consider the robust stability of the uncertain system.

Theorem 2 For given values of $h_{1}, h_{2}$ and $\mu$,System (5) is asymptotically stable, if there exist a scalar $\varepsilon_{i}>0, i=1,2$ and $\left[\begin{array}{ccc}P_{11} & P_{12} & P_{13} \\ * & P_{22} & P_{23} \\ * & * & P_{33}\end{array}\right]>0, Q_{j}>0, Z_{j}>0 j=1,2, S>0,\left[\begin{array}{cc}R_{11} & R_{12} \\ * & R_{22}\end{array}\right] \geq 0,\left[\begin{array}{cc}W_{11} & W_{12} \\ * & W_{22}\end{array}\right] \geq 0$

with appropriate dimensions such that the following LMIs hold

$$
\begin{aligned}
& {\left[\begin{array}{ccc}
\hat{\Phi}_{1} & \Gamma_{1}^{T} D & \varepsilon \Gamma_{2}^{T} \\
* & -\varepsilon I & 0 \\
* & * & -\varepsilon I
\end{array}\right]<0} \\
& {\left[\begin{array}{ccc}
\hat{\Phi}_{2} & \Gamma_{2}^{T} D & \varepsilon \Gamma_{2}^{T} \\
* & -\varepsilon I & 0 \\
* & * & -\varepsilon I
\end{array}\right]<0}
\end{aligned}
$$

Where

$$
\begin{aligned}
& \hat{\Phi}_{1}=\left[\begin{array}{cc}
\Phi+\Phi_{i} & T^{T} M_{1} \\
* & -M_{1}
\end{array}\right], i=1,2, \hat{\Phi}_{2}=\left[\begin{array}{cc}
\Phi+\Phi_{i} & T^{T} M_{2} \\
* & -M_{2}
\end{array}\right], i=1,2 \\
& \Gamma_{1}=\left[\begin{array}{llllllll}
P_{11}^{T} & 0 & 0 & 0 & P_{12}^{T} & P_{13}^{T} & M_{1}^{T}
\end{array}\right], \Gamma_{2}=\left[\begin{array}{lllllll}
E_{a} & 0 & E_{b} & 0 & 0 & 0 & 0
\end{array}\right], \Gamma_{3}=\left[\begin{array}{lllllll}
P_{11}^{T} & 0 & 0 & 0 & P_{12}^{T} & P_{13}^{T} & M_{2}^{T}
\end{array}\right] .
\end{aligned}
$$

Proof : replacing $A, B$ in (8),(9) with $A+\Delta A, B+\Delta B$, respectively, and using Lemma 4 completes the proof.

\section{Numerical examples}

In this section, we use two numerical examples to show that the proposed results are improvements over some exiting ones

Example 1. Consider nominal time-delay system with the following parameter

$$
A=\left[\begin{array}{cc}
-2 & 0 \\
0 & -0.9
\end{array}\right], B=\left[\begin{array}{cc}
-1 & 0 \\
-1 & -1
\end{array}\right]
$$

For given $\mu$ and unknown $\mu$,Table 1 provides the maximal allowable bounds of the delay $h_{2}$ for given lower bounds $h_{1}$. From the table, it is clear that the proposed stability is less conservative than those in $[2,10,12]$.

Example 2 Consider an uncertain system described by the following matrices

$$
A=\left[\begin{array}{cc}
-2+\delta_{1} & 0 \\
0 & -1+\delta_{2}
\end{array}\right], B=\left[\begin{array}{cc}
-1+\delta_{3} & 0 \\
-1 & -1+\delta_{4}
\end{array}\right]
$$

where, $\delta_{1}, \delta_{2}, \delta_{3}$ and $\delta_{4}$ unknown parameter, satisfy

$$
\left|\delta_{1}\right| \leq 1.6,\left|\delta_{2}\right| \leq 0.05,\left|\delta_{3}\right| \leq 0.1,\left|\delta_{4}\right| \leq 0.3
$$

When there is no restriction on the delay derivative, Table 2 shows the obtained maximum allowable delay bound for a varying $h_{1}$.From the table, it is clear to see that for this example, some exiting results have been improved.

Table 1 Maximum allowable delay bound $h_{2}$ for a given $h_{1}$ and $\mu$ 


\begin{tabular}{|c|c|c|c|c|c|c|}
\hline$\mu$ & ethod & $h_{1}=0$ & $h_{1}=1$ & $h_{1}=2$ & $h_{1}=3$ & $h_{1}=4$ \\
\hline \multirow{4}{*}{0.5} & [2] & 2.0439 & 2.0775 & 2.4404 & 3.2260 & 4.0649 \\
\hline & 10] & 2.0723 & 2.1276 & 2.5048 & 3.2591 & 4.0744 \\
\hline & 12] & 2.0801 & 2.1513 & 2.7113 & 3.3839 & 4.1136 \\
\hline & orem 1 & 2.2025 & 2.3912 & 2.9578 & 3.6384 & 4.3736 \\
\hline \multirow{4}{*}{0.9} & [2] & 1.3789 & 1.7424 & 2.4328 & 3.2234 & 4.0643 \\
\hline & 10] & 1.5304 & 1.8737 & 2.5048 & 3.2591 & 4.0744 \\
\hline & $12]$ & 1.6654 & 2.1251 & 2.7113 & 3.3839 & 4.1136 \\
\hline & orem 1 & 1.8299 & 2.7945 & 2.7945 & 3.4310 & 4.1280 \\
\hline \multirow{4}{*}{ Any $\mu$} & [2] & 1.3454 & 1.7424 & 2.4328 & 3.2234 & 4.0643 \\
\hline & 10] & 1.5296 & 1.8737 & 2.5048 & 3.2591 & 4.0744 \\
\hline & 12] & 1.6654 & 2.1251 & 2.7113 & 3.3839 & 4.1136 \\
\hline & orem 1 & 1.8299 & 2.7945 & 2.7945 & 3.4310 & 4.1280 \\
\hline \multicolumn{7}{|c|}{ Table 2 upper delay bound $h_{2}$ for given $h_{1}$} \\
\hline \multirow{2}{*}{ Method } & \multicolumn{6}{|c|}{$\overline{h_{1}}$} \\
\hline & 0 & 0.2 & 0.4 & 0.6 & 0.8 & 1.0 \\
\hline [11] & 0.9442 & 0.9757 & 1.0208 & 1.0795 & 1.1500 & 1.2308 \\
\hline$[13](\mathrm{N}=2)$ & 1.0571 & 1.0953 & 1.1385 & 1.1865 & 1.2392 & 1.2966 \\
\hline [9] & 1.1030 & 1.1337 & 1.1703 & 1.2123 & 1.2594 & 1.3111 \\
\hline Theorem 2 & 1.1420 & 1.1570 & 1.2073 & 1.2317 & 1.2680 & 1.3415 \\
\hline
\end{tabular}

\section{Conclusions}

This paper investigates the robust delay-dependent stability problem of a class of linear uncertain system with interval time-varying delay. A new L-K stability condition for this system was proposed. The key features of the stability condition include the delay-central point method for designing the L-K functional and a tighter integral inequality for bounding the cross-terms. As a result, less conservative results are achieved. Numerical examples have illustrated the effectiveness of the proposed method.

\section{References}

[1] Gu K, Kharitonov V L, Chen J. Stability of time-delay systems[M]. Basel: Birkhauser, 2003: $1-17$.

[2] He Y,Wang Q, Lin C, et al. Delay-range-dependent stability for systems with time-varying delay[J]. Automatica, 2007, 43(2):371-376.

[3] O.M. Kwon,M.J.Park,S.M. Lee.Augmented Lyapunov functional approach to stability of uncertain neutral systems with time-varying delays[J],Applied Mathematics and Computation,2009,207(1):202-212.

[4] O.M. Kwon, M.J.Park, S.M. Lee,E.J. Cha. Analysis on delay-dependent stability for neural networks with time-varying delays[J],Neurocomputing,2013,103: 114-120.

[5] Yue D, Tian D, Zhang Y. A piecewise analysis method to stability analysis of continuous/discrete systems with time-varying delay[J], International Journal Robust Nonlinear Control, 2009,19(13): 1493-1518.

[6] Wang C,Shen Y. Delay partitioning approach to robust stability analysis for uncertain stochastic systems with interval time-varying delay[J],IET Control Theory and Applications,2012,6(7):875-883. 
[7] Orihuela, L., Millan, P., Vivas, C., Rubio, F.R.Robust stability of nonlinear time-delay systems with interval time-varying delay[J],International Journal of Robust and Nonlinear Control ,2011,21(7):709-724.

[8] Zhang X-M ,Han Q-L .A delay decomposition approach to delay-dependent stability for linear systems with time-varying delays[J],International Journal of Robust and Nonlinear Control,2009,19(17):1922-1930.

[9] K. Ramakrishnan, G. Ray .Robust stability criteria for uncertain linear systems with interval time-varying delay [J],Journal of Control Theory and Applications , 2011, 9 (4):559-566.

[10] Shao H-Y. New delay-dependent stability criteria for systems with interval delay [J]. Automatica, 2009,45(3):744-749.

[11]Jiang X-F , Han Q-L. New stability criteria for linear systems with interval time varying delay[J], Automatica, 2008, 44(10):2680 -2685.

[12]C. Peng, Y.C.Tian. Improved delay-dependent robust stability criteria for uncertain systems with interval time-varying delay[J]. IET Control Theory and Application, 2008,2(9)9:752-761,

[13]K. Ramakrishnan, G. Ray. Delay-dependent robust stability criteria for linear uncertain systems with interval time varying delay[C].TENCON 2009-2009 IEEE Region 10 Conference. Singapore: IEEE, 2009: 5396259.

[14]Petersen I R, Hollot C V. A Riccati equation approach to the stabilization of uncertain linear systems[J]. Automatica, 1986,22(4): 397-411. 